\title{
Response to Comment on: Elias et al. Adipose Tissue Overexpression of Vascular Endothelial Growth Factor Protects Against Diet-Induced Obesity and Insulin Resistance. Diabetes 2012;61:1801-1813
}

Ivet Elias, ${ }^{1,2,3}$ Sylvie Franckhauser, ${ }^{1,3}$ and Fatima Bosch ${ }^{1,2,3}$

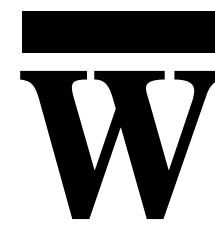

e appreciate the interest of Drs. Lu and Zheng (1) in our work showing that adipose tissue overexpression of vascular endothelial growth factor (VEGF) in transgenic mice protects against diet-induced obesity and insulin resistance (2). We agree that changes in VEGF expression may also play a role in the development of diseases such as obesity, diabetes, or cancer and that the underlying mechanisms are complex, as reflected by recent literature. Indeed, Lu et al. (3) reported that repression of VEGF-A, accompanied by an increase in VEGF-B, leads to protection against high-fat diet-induced obesity, similarly to what we and others have observed with VEGF-A overexpression $(2,4)$. The fact that both repression and overexpression of VEGF-A results in the same effect demonstrates the complexity of the system. In accordance with our results, a recent article from Sun et al. (4) also shows a protective effect of VEGF-A-inducible overexpression in adipose tissue against diet-induced obesity and insulin resistance. However, unlike in our model, inducible VEGF-A overexpression exerts its effects mainly on white adipose tissue (WAT) (4), similarly to conditional VEGF repression in adipose tissue (3). In these two models, a brown adipose tissue (BAT)-like phenotype was observed in WAT. In contrast, in our transgenic mice, BAT-specific markers were not detected in WAT, but an enhancement of BAT thermogenesis (supported by increased UCP1 and PGC1- $\alpha$ BAT protein levels) was observed (2). All these results clearly highlight a potential role of VEGF in BAT differentiation and function. Supporting the observation that VEGF may control cell differentiation, it has recently been demonstrated that VEGF suppresses adipocyte differentiation independently of VEGF receptor signaling activation (5). This also points out that VEGF can act through a wide range of mechanisms. Therefore a better understanding of VEGF actions on metabolism would be crucial for the treatment of obesity, insulin resistance, and other diseases.

\section{ACKNOWLEDGMENTS}

No potential conflicts of interest relevant to this article were reported.

\section{REFERENCES}

1. Lu X, Zheng Y. Comment on: Elias et al. Adipose tissue overexpression of vascular endothelial growth factor protects against diet-induced obesity and insulin resistance. Diabetes 2012;61:1801-1813 (Letter). Diabetes 2013;62: e3. DOI: $10.2337 / \mathrm{db} 12-1130$

2. Elias I, Franckhauser S, Ferré T, et al. Adipose tissue overexpression of vascular endothelial growth factor protects against diet-induced obesity and insulin resistance. Diabetes 2012;61:1801-1813

3. Lu X, Ji Y, Zhang L, et al. Resistance to obesity by repression of VEGF gene expression through induction of brown-like adipocyte differentiation. Endocrinology 2012;153:3123-3132

4. Sun K, Wernstedt Asterholm I, Kusminski CM, et al. Dichotomous effects of VEGF-A on adipose tissue dysfunction. Proc Natl Acad Sci USA 2012;109: 5874-5879

5. Liu Y, Berendsen AD, Jia S, et al. Intracellular VEGF regulates the balance between osteoblast and adipocyte differentiation. J Clin Invest 2012;122: 3101-3113

\footnotetext{
From the ${ }^{1}$ Center of Animal Biotechnology and Gene Therapy, Universitat Autònoma de Barcelona, Barcelona, Spain; the ${ }^{2}$ Departments of Biochemistry and Molecular Biology, School of Veterinary Medicine, Universitat Autònoma de Barcelona, Barcelona, Spain; and the ${ }^{3}$ CIBER de Diabetes y Enfermedades Metabólicas Asociadas (CIBERDEM), Barcelona, Spain. Corresponding author: Fatima Bosch, fatima.bosch@uab.es.

DOI: $10.2337 / \mathrm{db} 12-1274$

C 2013 by the American Diabetes Association. Readers may use this article as long as the work is properly cited, the use is educational and not for profit, and the work is not altered. See http://creativecommons.org/licenses/by -nc-nd/3.0/ for details.
} 